\title{
Comment on "Generalization of the Kohn-Sham system that can represent arbitrary one-electron density matrices"
}

\author{
Mario Piris ${ }^{1,2}$ and Katarzyna Pernal $^{3}$ \\ ${ }^{1}$ Kimika Fakultatea, Euskal Herriko Unibertsitatea (UPV/EHU), \\ and Donostia International Physics Center (DIPC), 20018 Donostia, Euskadi, Spain. \\ ${ }^{2}$ IKERBASQUE, Basque Foundation for Science, 48013 Bilbao, Euskadi, Spain. and \\ ${ }^{3}$ Institute of Physics, Lodz University of Technology, \\ ul. Wolczanska 219, 90-924 Lodz, Poland.
}

\begin{abstract}
Hubertus J. J. van Dam [Phys. Rev. A 93, 052512, 2016] claims that the one-particle reduced density matrix (1RDM) of an interacting system can be represented by means of a single-determinant wavefunction of fictitious non-interacting particles. van Dam introduced orbitals within a mean-field framework that produce energy levels similar to Hartree-Fock (HF) orbital energies, therefore he also claims that conventional analyses based on Koopmans theorem are possible in 1RDM functional theory (1RDMFT). In this comment, we demonstrate that both claims are unfounded.
\end{abstract}

Inspired by the Kohn-Sham [1] practical approach of the density functional theory (DFT), Hubertus J. J. van Dam has introduced [2] a new kind of generalized orbitals $\left\{\mathbf{G}_{s}\right\}$ to define an auxiliary Slater determinant in the one-particle reduced density matrix (1RDM) functional theory (1RDMFT) 3]. An alternative derivation of such a system of noninteracting particles was also put forward by Requist and Pankratov [4]. In contrast to latter formulation, which resorts to an ensemble of degenerate Slater determinants, i.e., a mixed state, van Dam presented a detailed analysis of how a Slater determinant can generate any $1 \mathrm{RDM}$ of an interacting system. van Dam proposes to parametrize the occupation numbers $\left\{n_{i}\right\}$ by means of a set of $n_{b}$ orthonormal vectors $\left\{\mathbf{C}_{r}\right\}$ (Eq. (5) of [2]):

$$
\mathbf{C}_{r} \mathbf{C}_{s}=\sum_{i=1}^{n_{b}} C_{i r}^{*} C_{i s}=\delta_{r s}
$$

The columns of the matrix $\mathbf{C}$ are referred to as correlation functions. In a system with $n_{e}$ electrons $\left(n_{e}<n_{b}\right)$ of a given spin, there are $n_{e}$ occupied correlation functions and all others are unoccupied. Accordingly, the occupation numbers are given by

$$
n_{i}=\sum_{r=1}^{n_{e}} C_{i r}^{*} C_{i r}=\sum_{r=1}^{n_{e}}\left|C_{i r}\right|^{2}
$$

Instead of working with $\left\{n_{i}\right\}$, imposing the $n_{e^{-}}$ representability conditions on them, van Dam proposes to replace them by the set of vectors $\left\{\mathbf{C}_{r}\right\}$. With the above definitions a new set of $n_{b}$ orthonormal generalized orbitals $\left\{\mathbf{G}_{s}\right\}$ are defined, where every vector $\mathbf{G}_{s}$ is expanded as (Eq. (7) of [2])

$$
G_{s}(r)=\sum_{a, i=1}^{n_{b}} N_{a i} C_{i s} \chi_{a}(r)
$$

In Eq. (3), $\chi_{a}(r)$ represents the basis functions and $\left\{\mathbf{N}_{i}\right\}$ is the orthonormal set of natural orbitals. Considering the generalized orbitals, van Dam defines a density matrix $\mathbf{D}$, but unfortunately he made a mistake in the calculation of its matrix elements, namely, the Eq. (16) of Ref. [2] is not correct. Indeed, the probability density given a vector $\mathbf{G}_{s}$ according to Eq. (15) of Ref. [2] is

$$
\begin{aligned}
G_{s}(r) G_{s}^{*}\left(r^{\prime}\right) & =\left(\sum_{a, i=1}^{n_{b}} N_{a i} C_{i s} \chi_{a}(r)\right)\left(\sum_{b, j=1}^{n_{b}} N_{b j} C_{j s} \chi_{b}\left(r^{\prime}\right)\right)^{*} \\
& =\sum_{a, b=1}^{n_{b}} \chi_{a}(r) D_{a b}^{s} \chi_{b}^{*}\left(r^{\prime}\right)
\end{aligned}
$$

where

$$
D_{a b}^{s}=\sum_{i, j=1}^{n_{b}} N_{a i} C_{i s} N_{b j}^{*} C_{j s}^{*}
$$

which is different from Eq. (16) of Ref. [2]. Note that only a summation with respect to index $i$ appears in Eq. (16), instead of the two summations with respect to $i$ and $j$ of equation (4), respectively. This implies that the results derived subsequently are also not valid. Particularly, the 1RDM obtained from the Slater determinant wavefunction, Eq. (17) of Ref. [2], should read (compare with Eq. (18) of Ref. [2]):

$$
\sum_{s=1}^{n_{e}} G_{s}(r) G_{s}^{*}\left(r^{\prime}\right)=\sum_{a, b=1}^{n_{b}} \chi_{a}(r) D_{a b} \chi_{b}^{*}\left(r^{\prime}\right)
$$

where

$$
D_{a b}=\sum_{i, j=1}^{n_{b}} N_{a i}\left(\sum_{s=1}^{n_{e}} C_{i s} C_{j s}^{*}\right) N_{b j}^{*}
$$

contrary to what Eq. (20) of [2] shows, namely,

$$
d_{i j}=\sum_{s=1}^{n_{e}} C_{i s} C_{j s}^{*} \neq \sum_{s=1}^{n_{e}} C_{i s} C_{i s}^{*}=d_{i}
$$

The Eqs. (7) and (8) imply that the natural orbitals $\left\{\mathbf{N}_{i}\right\}$ are not the eigenfunctions of the 1RDM obtained 
from the determinant wavefunction, as expected. The genuine $1 \mathrm{RDM}$ has a unique set $\left\{\mathbf{G}_{s}\right\}$ of eigenfunctions with occupations numbers 0 and 1 . The set is unique up to the rotations among degenerate orbitals. Such a matrix is idempotent, whereas the 1RDM of an interacting system is never idempotent. The noninteracting system proposed by van Dam cannot represent the 1-RDM of any system with fractional occupation numbers.

In order to implement the auxiliary wavefunction, an optimization scheme was devised based on an explicit energy functional of the 1RDM in both spin channels (Eq. (22) of Ref. [2]). This assumption was addressed at the early stage of the 1RDMFT development [5, 6], and recently by Pernal [7] and Piris and Ugalde [8]. Unfortunately, apart from the special case of the Hartree-Fock (HF) energy expression that may be viewed as the simplest 1RDM functional, none of the currently known functionals is explicitly given in terms of the 1RDM, including the accurate functional describing two-electron closed-shell systems [9], namely,

$$
\begin{gathered}
E\left(2 e^{-}\right)=2 \sum_{p=1}^{\infty} n_{p} \mathcal{H}_{p p}+n_{1} \mathcal{L}_{11} \\
+\sum_{p, q=2}^{\infty} \sqrt{n_{q} n_{p}} \mathcal{L}_{p q}-2 \sum_{p=2}^{\infty} \sqrt{n_{1} n_{p}} \mathcal{L}_{p 1}
\end{gathered}
$$

where $n_{p}$ denotes the occupation number in the spatial orbital $p, \mathcal{H}_{p p}$ is the one-electron matrix elements of the core-Hamiltonian, and $\mathcal{L}_{p q}=\langle p p \mid q q\rangle$ is the exchange and time-inversion integral [10]. The natural orbital functional (NOF) (9) is obtained from the exact wavefunction [11, 12] assuming that all natural occupation amplitudes, with the exception of the first one, are negative if the first amplitude is chosen to be positive 9]. It can be readily seen that the electron-electron interaction energy cannot be explicitly expressed in terms of the 1-RDM due to the different phase factors $( \pm 1)$ of the occupation amplitudes. In the case of $N$ electrons, a generalization of this functional is the extended version of PNOF5 [13], which can be obtained from a wavefunction of an antisymmetrized product of strongly orthogonal geminals (APSG) [14]. van Dam claims that a wide range of energy expressions encompasses the explicit dependence on the 1RDM, but this is unfounded.

The functionals currently in use are only known in the basis where the 1RDM is diagonal. This implies that they are not functionals explicitly dependent on the $1 \mathrm{RDM}$ and retain some dependence on the twoparticle reduced density matrix (2RDM). For this reason, it is more appropriate to speak of a NOF rather than a functional of the 1RDM for an approximate functional. In this vein, in the NOF theory (NOFT), the natural orbitals (NOs) are the orbitals that diagonalize the 1RDM corresponding to an approximate expression of the energy, like those obtained from an approximate wavefunction.

In NOFT, functionals still depend explicitly on the 2RDM, hence the energy is not invariant with respect to a unitary transformation of the orbitals. Consequently, the orbital optimization cannot be reduced to a pseudo-eigenvalue problem considering the diagonal representation of the matrix of Lagrange multipliers, associated with the orbital orthonormality conditions. On this issue, Löwdin already drew attention in his 1955 landmark paper [15]. Only if the electronelectron interaction energy is an explicit functional of the 1RDM, the functional derivative present in Eq. (29) of Ref. [2] may be directly calculated [5]. In the case of an implicit functional, the proper procedure to obtain the derivative was proposed by Pernal [7], which is based on using the chain rule and first-order perturbation theory applied to the eigenequation of the 1RDM. The van Dam's assertion that the secular equation to determine the NOs (Eq. (30) of Ref. [2]) is the same as the KS equation is not applicable in NOFT. Even for the yet unknown exact groundstate functional of the 1RDM, the secular equation to determine the NOs bears solely a striking formal resemblance to the HF equations [5].

The exact functional of closed-shell two-electron systems given by the Eq. (9), as well as PNOF5, are approximate but are strictly N-representable functionals. This points to another issue, that of the N-representability problem of the functional [16 18]. The latter refers to the conditions that guarantee the one-to-one correspondence between $E[\Psi] \equiv E[2 \mathrm{RDM}]$ and $E[1 \mathrm{RDM}]$. Several proposals have appeared in the literature [19, 20], in which the 2RDM is expressed in terms of the $1 \mathrm{RDM}$ by means of a reconstruction functional. Accordingly, these reconstructions must comply with the known conditions for the N-representability of the 2RDM. It has been generally assumed that there is no N-representability problem of the functional, as it was believed that only N-representable conditions on the 1-RDM were necessary. The ensemble N-representability constraints for acceptable 1RDMs are easy to implement, but are insufficient to guarantee that the reconstructed 2RDM is N-representable, and thereby the functional either.

The first explicit approximate relation between 2RDM and $1 R D M$ containing one free parameter was that proposed by Müller in 1984 [21], generalized later by Sharma et al. 22] as the power functional, and applied in work [2] to Be and LiH. While it is true that the Müller functional has a simple dependence upon the 1RDM, it has serious shortcomings [9]. The matter is that this simple JK-only functional avoids the phase dilemma discussed above, that stems from the fact that the construction of the functional requires a choice over a large number of possible combinations signs in the electron-electron interaction between NOs. In doing so, the functional seems to depend properly on the 1RDM, but violates the N-representability conditions for the 2RDM. Several reconstruction functionals for NOFT, including Müller's, were investigated by Herbert and Harriman [16], and illustrative calculations were precisely made for Be and LiH. They documented extensive Nrepresentability violations for proposed reconstruction functionals. Although we can obtain quite reasonable results, these do not guarantee that calculations made 
using the power functional are accurate.

Finally, the explicit dependence on the 2RDM in NOFT leads to two other important consequences. Firstly, the correct procedure in NOFT for describing the electron detachment in terms of one-electron quantities is the use of the extended Koopmans' theorem, which provides the connection between the $1 \mathrm{RDM}$ and 2RDM with ionization potentials [23 25]. Secondly, the NOFT provides two complementary representations of the one-electron picture, namely, the NO representation and the canonical orbital (CO) representation [26]. The former arises directly from the optimization process solving the corresponding Euler equations, whereas the latter is attained from the diagonalization of the matrix of Lagrange multipliers obtained in the natural orbital representation. The
1-RDM is diagonal in the NO representation but not the Lagrangian, which is only a Hermitian matrix. Conversely, in the CO representation, the Lagrangian is diagonal but not the $1 \mathrm{RDM}$. It has been shown [26] by means of the extended Koopmans' theorem that the one-particle energies associated to the COs can yield the ionization potentials when the 1RDM remains close to the diagonal form.

To summarize, the procedure proposed by van Dam for obtaining a noninteracting system that can represent the one-electron density matrix of any system, as well as the conventional analysis based on Koopmans' theorem, are not valid.

Financial support comes from the Spanish MINECO/FEDER Project No. CTQ2015-67608-P.
[1] W. Kohn and L. Sham, Phys. Rev. 140, A1133 (1965).

[2] H. J. J. van Dam, Phys. Rev. A 93, 052512 (2016).

[3] T. L. Gilbert, Phys. Rev. B 12, 2111 (1975).

[4] R. Requist and O. Pankratov, Phys. Rev. B 77, 235121 (2008).

[5] R. A. Donnelly, J. Chem. Phys. 71, 2874 (1979).

[6] T. T. Nguyen-Dang, E. V. Ludeña, and Y. Tal, J. Mol. Struc.: THEOCHEM 120, 247 (1985).

[7] K. Pernal, Phys. Rev. Lett. 94, 233002 (2005).

[8] M. Piris and J. M. Ugalde, J. Comput. Chem. 30, 2078 (2009).

[9] S. Goedecker and C. J. Umrigar, in Many-electron densities and reduced density matrices, edited by J. Cioslowski (Kluwer Academic/Plenum Publishers, New York, 2000), pp. 165-181.

[10] M. Piris, J. Math. Chem. 25, 47 (1999).

[11] H. Shull and P. O. Löwdin, J. Chem. Phys. 30, 617 (1959).

[12] W. Kutzelnigg, Theoret. chim. Acta (Berl.) 1, 327 (1963).

[13] M. Piris, J. M. Matxain, and X. Lopez, J. Chem. Phys. 139, 234109 (2013).

[14] K. Pernal, Comp. Theor. Chem. 1003, 127 (2013).

[15] P. O. Lowdin, Phys. Rev. 97, 1474 (1955).

[16] J. M. Herbert and J. E. Harriman, Journal of Chem- ical Physics 118, 10835 (2003).

[17] M. Piris, J. M. Matxain, X. Lopez, and J. M. Ugalde, J. Chem. Phys. 133, 111101 (2010).

[18] E. V. Ludeña, F. J. Torres, and C. Costa, J. Mod. Phys. 04, 391 (2013).

[19] M. Piris, in Reduced-Density-Matrix Mechanics: with applications to many-electron atoms and molecules, edited by D. A. Mazziotti (John Wiley and Sons, Hoboken, New Jersey, USA, 2007), chap. 14, pp. 387-427.

[20] K. Pernal and K. J. H. Giesbertz, Top Curr Chem 368, 125 (2016).

[21] A. M. K. Muller, Phys. Lett. 105A, 446 (1984).

[22] S. Sharma, J. K. Dewhurst, N. N. Lathiotakis, and E. K. U. Gross, Phys. Rev. B 78, 201103(R) (2008).

[23] K. Pernal and J. Cioslowski, Chem. Phys. Lett. 412, 71 (2005).

[24] P. Leiva and M. Piris, J. Mol. Struc.: THEOCHEM 770, 45 (2006).

[25] M. Piris, J. M. Matxain, X. Lopez, and J. M. Ugalde, Journal of Chemical Physics 136 (2012).

[26] M. Piris, J. M. Matxain, X. Lopez, and J. M. Ugalde, Theor. Chem. Acc. 132, 1298 (2013). 